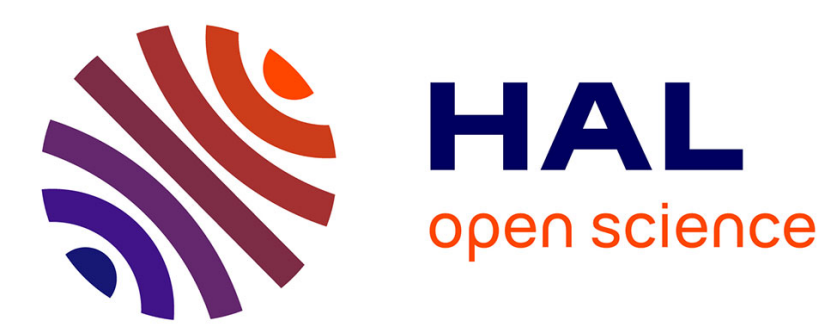

\title{
Visualization of surface acoustic waves by scanning electron microscopy
}

D. Roshchupkin, M. Brunel, L. Tucoulou

\section{To cite this version:}

D. Roshchupkin, M. Brunel, L. Tucoulou. Visualization of surface acoustic waves by scanning electron microscopy. Journal de Physique IV Proceedings, 1994, 04 (C5), pp.C5-1229-C5-1232. 10.1051/jp4:19945272 . jpa-00252963

\section{HAL Id: jpa-00252963 https://hal.science/jpa-00252963}

Submitted on 1 Jan 1994

HAL is a multi-disciplinary open access archive for the deposit and dissemination of scientific research documents, whether they are published or not. The documents may come from teaching and research institutions in France or abroad, or from public or private research centers.
L'archive ouverte pluridisciplinaire HAL, est destinée au dépôt et à la diffusion de documents scientifiques de niveau recherche, publiés ou non, émanant des établissements d'enseignement et de recherche français ou étrangers, des laboratoires publics ou privés. 


\title{
Visualization of surface acoustic waves by scanning electron microscopy
}

\author{
D.V. ROSHCHUPKIN ${ }^{* * * *}$, M. BRUNEL ${ }^{* *}$ and R. TUCOULOU**
}

* Institute of Microelectronic Technology, Russian Academy of Sciences, 142432 Chernogolovka, Moscow District, Russia

${ }^{* *}$ CNRS, Laboratoire de Cristallographie, BP. 166X, 38042 Grenoble cedex, France

résumé : This paper reports a scanning electron microscopy visualization of surface acoustic waves in single- and poly-domains $\mathrm{LiNbO}_{3}$ crystals. This SEM method is very useful to visualize traveling and standing surface acoustic waves in SAW-devices and to observe the interaction between SAWs and defects on crystal surfaces. Furthermore, we investigated the interaction between SAWs and regular domain structures in $\mathrm{LiNbO}_{3}$ crystals.

\section{Introduction}

Today, the scanning electron microscopy (SEM) is the best method for the observation of surface and bulk acoustic waves propagation in piezoelectric and ferroelectric materials [1-6]. To visualize the SAW propagation, two different modes are available. The first one, called stroboscopic mode, is based on the high-frequency modulation of the electron beam by the frequency of the traveling SAW. Thus, the electron probe is in phase synchronization with the traveling SAW and the secondary electron emission from the crystal surface is proportional to the electric field which accompanies the propagation of the traveling SAW [1-4]. In the second mode, which is more handy, a high-frequency modulation of the low energy secondary electrons (with energy $\sim 1-3 \mathrm{eV}$ ) is performed by the stationary electrostatic interference field formed above the crystal surface by the interaction between the varying electric field of the SAW and the component, normal to the crystal surface, of the electromagnetic radiation field of the interdigital transducer (IDT). Since they are excited by the same source (IDT) and with the same frequency, the electromagnetic and acoustic waves are mutually coherent. Since the wavelength of the electromagnetic wave is larger than the wavelength of the SAW, the period of the stationary interference field is equal to the period of the SAW. Thus, the low energy secondary electrons are modulated by the stationary electric interference field [5-6]. In our investigations we used this second method.

We also studied the interaction between SAWs and regular domain structures in $\mathrm{LiNbO}_{3}$ crystals, which is interesting for the elaboration of new devices based on surface acoustic waves.

\section{Experimental results}

These experiments were performed at the Centre National de la Recherche Scientifique, Grenoble, using a JEOL JSM-840 SEM with an accelerating voltage $E_{0}=1-3 \mathrm{keV}$ and a probe current $I_{0}=10^{-10} \mathrm{~A}$. The use of higher accelerating voltages is not possible because the insulating ferroelectric $\mathrm{LiNbO}_{3}$ substrate would become highly charged and thus bring about a distortion of the image because of the deflection of the electron probe. Fig. 1(a) shows the SEM image of a traveling SAW $(\Lambda=30 \mu \mathrm{m})$, which was excited by an ordinary IDT at its resonance excitation frequency $f_{0}=116 \mathrm{MHz}$. The SAW propagates along the $Z$ axis with a velocity $V=3.488 \mathrm{~km} / \mathrm{s}$. The contrast of the SEM image is due to the opposite potentials of the maxima and the minima of the traveling SAW. 

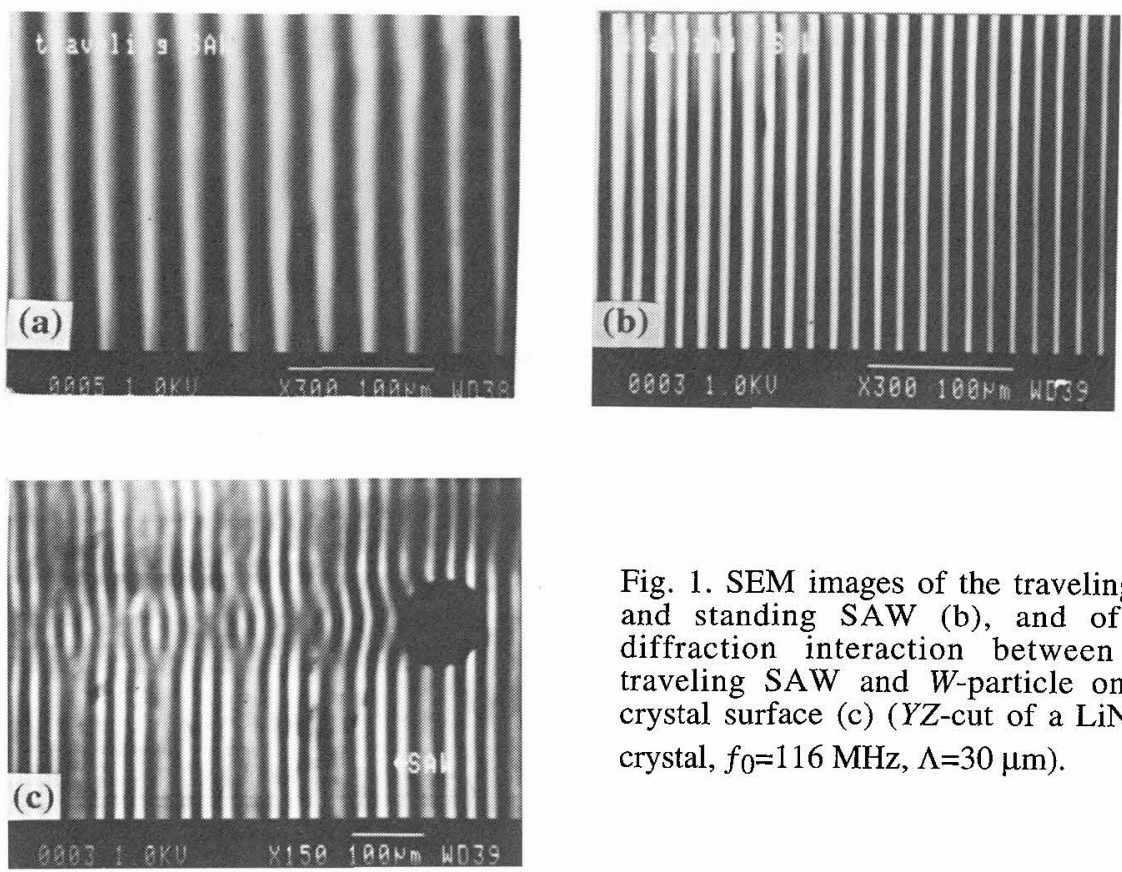

Fig. 1. SEM images of the traveling (a) and standing SAW (b), and of the diffraction interaction between the traveling SAW and $W$-particle on the crystal surface (c) (YZ-cut of a $\mathrm{LiNbO}_{3}$ crystal, $\left.f_{0}=116 \mathrm{MHz}, \Lambda=30 \mu \mathrm{m}\right)$.

The positive potential decreases the number of detected secondary low energy electrons from the crystal surface and gives a dark contrast, while the negative potential increases the number of detected secondary electrons and gives a bright contrast. The period of the voltage contrast of the SEM image of the traveling SAW is equal to the SAW wavelength, $\Lambda=30 \mu \mathrm{m}$. Furthermore, it is possible to observe standing SAWs with the SEM. Fig. 1(b) is a photograph of a standing SAW which was formed by interaction between two counter-propagating traveling SAWs, excited at the resonance excitation frequency $f_{0}=116 \mathrm{MHz}$. There is a voltage contrast between the nodes and antinodes of the standing SAW. Fig. 1(c) shows the interaction between the traveling SAW and a circular $W$-particle with a diameter of $100 \mu \mathrm{m}$. One can notice that after the circular $W$-particle, a diffraction pattern is formed in the acoustic beam. This effect is due to the diffraction of the SAW on the particle and to the decrease of the SAW velocity under the $W$-particle.

Today, the development of new types of SAW-devices [5-6] based on the regular domain structures in ferroelectric crystals is very interesting for acoustoelectronics. It is indeed possible to use regular domain structures in ferroelectric $\mathrm{LiNbO}_{3}$ crystals for the excitation of SAWs by piezoelectric effect and for the reflection of SAWs on domain walls. In order to investigate these phenomena, we used a $\mathrm{LiNbO}_{3}$ crystal in which the regular domain structures were formed by the method of aftergrowth thermoelectrical treatment near the Curie temperature $\left(T_{\mathrm{c}} \sim 1100^{\circ} \mathrm{C}\right)$ at the conditions of temperature gradient and electric field [7]. Fig. 2(a) shows the experimental scheme of the SAW excitation by a curved regular domain structure in aYZ-cut of a $\mathrm{LiNbO}_{3}$ crystal. The curved regular domain structure with domain width $\sim 70 \mu \mathrm{m}$ was only formed in one part of the crystal. The radius of curvature of the domain structure was $16 \mathrm{~mm}$. Two $A u$-electrodes with thickness $\sim 1000 \AA$ were evaporated on the both sides of the domain structure (XZ-plane) for the SAW excitation. 

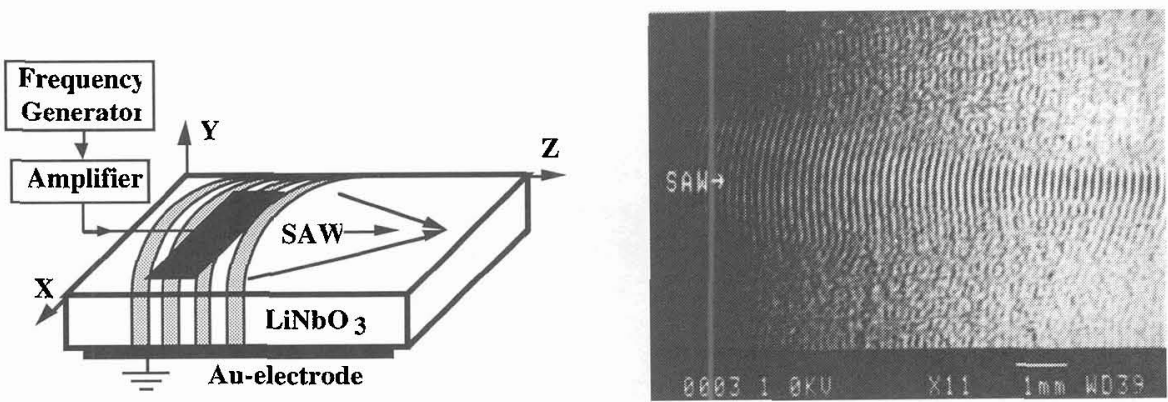

(b)

Fig. 2. Excitation of the SAWs by the regular domain structure: (a) experimental scheme, (b) SEM image of the SAWs excited by the curved regular domain structure.

The tensor of the piezoelectric coefficient of the $\mathrm{LiNbO}_{3}$ crystal $d_{i j}$,

$$
d_{i j}=\left[\begin{array}{cccccc}
0 & 0 & 0 & 0 & d_{15} & -d_{22} \\
-d_{22} & d_{22} & 0 & d_{15} & 0 & 0 \\
d_{31} & d_{31} & d_{33} & 0 & 0 & 0
\end{array}\right],
$$

prooves that the electric field $E_{2}$ applied to the $A u$-electrodes along the piezoelectric axis $Y$ excites some mechanical strains in this direction through the longitudinal piezoelectric effect $\left(r_{2}=d_{22} \cdot E_{2}\right)$. Since neighboring domains have opposite polarizations, the electric field $E_{2}$ excites a traveling SAW by the squeezing of one domain and the stretching of the next one. The traveling SAW propagates in the YZ-cut of the crystal along the $Z$ axis with the velocity $V=3.488 \mathrm{~km} / \mathrm{s}$. Photograph $2(\mathrm{~b})$ is the SEM image of a SAW excited by the longitudinal piezoelectric effect by applying the high-frequency electric field $E_{2}$ with an amplitude of the input signal on the $A u$-electrodes $U=12 \mathrm{~V}$ and at the resonance excitation frequency $f_{0}=25 \mathrm{MHz}$. The wavelength of the $\operatorname{SAW}(\Lambda=140 \mu \mathrm{m})$, corresponds to the width of two domains. The curved regular domain structure excites the SAW with a curved wave front which leads to focus the SAW to a narrow beam in the focal point. The focal point is placed at $16 \mathrm{~mm}$ from the curved regular domain structure. The focalization of the acoustic beam leads to increase the amplitude of the SAW in the focal point. Fig. 2(b) demonstrates that the maximum of the voltage contrast of the image is obtained in the focal point. It corresponds to the maximum of amplitude of the SAW. Right after the focal point, the acoustic beam begins to diverge, and the wave front of the SAW has a reversed curvature. Thus, we have shown that regular domain structures in ferroelectric crystals can be used for the excitation of traveling SAWs through the piezoelectric effect, as an ordinary interdigital transducer.

Another interesting possibility is the SAW propagation across a regular domain structure. Fig. 3 shows the experimental scheme (a) and the SEM image of the SAW propagation across the domain wall $\mathrm{AB}$ (b) in the $Y Z$-cut of a $\mathrm{LiNbO}_{3}$ crystal. The SAW with a wavelength of $30 \mu \mathrm{m}$, is excited by an IDT with a resonance excitation frequency $f_{0}=116 \mathrm{MHz}$ and propagates along the $Z$ axis. On the fig. 3(b), the $\mathrm{SAW}$ inverts the voltage contrast of the SEM image on the domain wall AB. This is due to the opposite directions of the piezoelectric axis $Y$ in the neighboring domains.
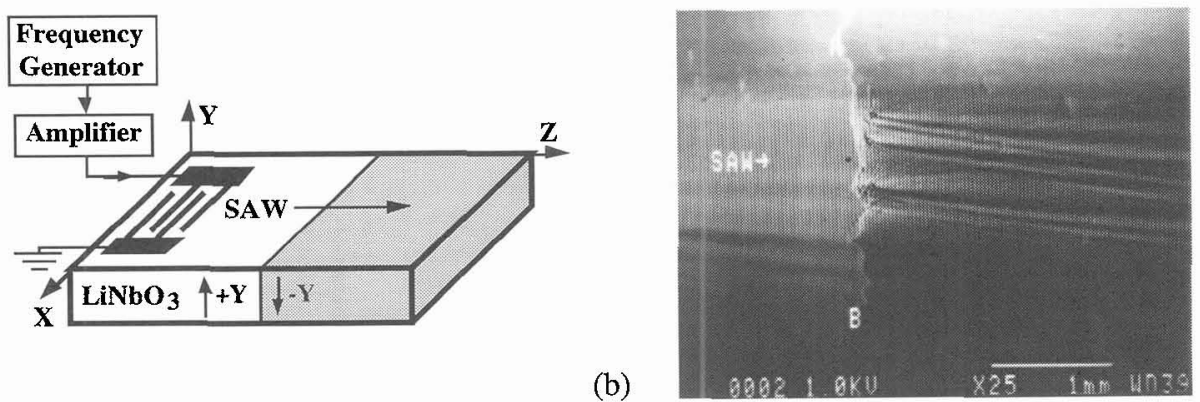

(b) 
Fig. 3. SAW propagation across the regular domain structure: (a) experimental scheme, (b) SEM image of the SAWs (AB - domain wall).

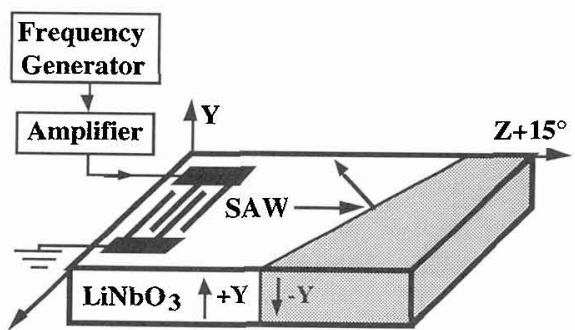

(a)

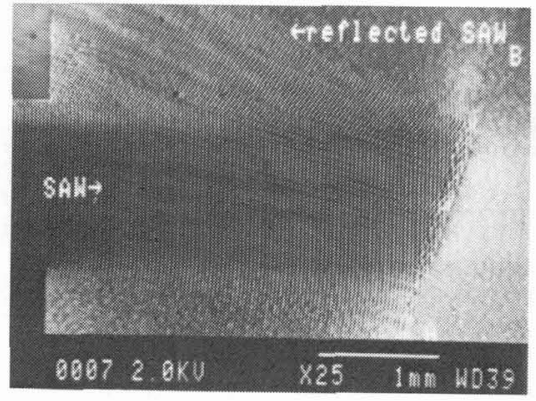

(v)

Fig. 4. SAW reflection on the domain wall: (a) experimental scheme, (b) SEM image of the $S A W$ reflection $(\mathrm{AB}$ - domain wall).

One can notice that the SAW diffracts on the roughness of the domain wall AB, which induces some modifications in the propagation of the energy in the acoustic beam after the domain wall $\mathrm{AB}$. The experimental scheme is shown in Fig. 4 (a) and Fig. 4 (b) is the SEM image of the SAW reflection on a domain wall in the $Y Z$-cut of a $\mathrm{LiNbO}_{3}$ crystal. The SAW with a wavelength of $30 \mu \mathrm{m}$ propagates along the $\mathrm{Z}+15^{\circ}$ direction. The angle between the direction of the SAW propagation and the domain wall $A B$ is $75^{\circ}$. It is seen from the SEM image of fig. $4(\mathrm{~b})$ that the SAW reflects on the domain wall AB. The incident and reflected angles are equal.

\section{Conclusion}

This paper demonstrates the real possibility to use the regular domain structures in ferroelectric crystals for excitation and reflection of SAWs.

\section{References}

[1] G. Eberharter and H.P. Feuerbaum, Appl. Phys. Lett. 37, 698 (1980).

[2] H.P. Feuerbaum, G. Eberharter, and G. Tobolka, Scanning Electron Microsc. 1, 503 (1980).

[3] N.D. Wittels and W.J. Tanski, Scanning Electron Microsc. 1, 319 (1980).

[4] H. Bahadur and R. Parshad, Scanning Electron Microsc. 1, 509 (1980).

[5] D.V. Roshchupkin, Th. Fournier, M. Brunel, O.A. Plotitsyna, and N.G. Sorokin, Appl. Phys. Lett. 60, 2330 (1992).

[6] D.V. Roshchupkin, Th. Fournier, M. Brunel, O.A. Plotitsyna, and N.G. Sorokin, Scanning Microscopy 6, 993 (1992).

[7] V.V. Antipov, A.A. Blistanov, N.G. Sorokin, and S.I. Chizhikov, Sov. Phys. Crystallogr. 30, 428 (1985). 